\title{
FIXED-POINT THEOREMS FOR CERTAIN CLASSES OF NONEXPANSIVE MAPPINGS ${ }^{1}$
}

\author{
L. P. BELLUCE AND W. A. KIRK
}

1. Introduction. A mapping $f$ of a metric space $M$ into itself is called nonexpansive if $d(f(x), f(y)) \leqq d(x, y)$ for each $x, y \in M$. For each $x \in M$, let $O\left(f^{n}(x)\right)$ denote the sequence of iterates of $f^{n}(x)$, that is,

$$
O\left(f^{n}(x)\right)=\bigcup_{i=n}^{\infty}\left\{f^{i}(x)\right\}, \quad n=0,1,2, \cdots,
$$

where it is understood that $f^{0}(x)=x$. Our main purpose here is to prove fixed-point theorems for nonexpansive mappings $f$ for which the diameters of the sets $O\left(f^{n}(x)\right)$ satisfy a condition introduced below, a condition which is suggested by a consideration of the Banach Contraction Principle. For such mappings $f$, compactness of $M$ is seen to imply that every sequence of iterates $\left\{f^{n}(x)\right\}$ of $x$ converges to a fixed-point of $f$ (which is not necessarily unique) while if $M$ is a weakly compact, closed, and convex subset of a Banach space, then the existence of a fixed-point for $f$ is established. In the final section we show how the results of this paper lead in an indirect way to a generalization of Theorem 3 of [1].

2. Limiting orbital diameters. For a subset $A$ of $\mathrm{M}$, let $\delta(A)$ $=\sup \{d(x, y): x, y \in A\}$ denote the diameter of $A$, and let $f: M \rightarrow M$.

In general the sequence $\delta\left(O\left(f^{n}(x)\right)\right)$ is nonincreasing and has limit $r(x) \geqq 0$. We call the number $r(x)$ (which may be infinite) the limiting orbital diameter of $f$ at $x$, and introduce the following definition:

Definition. If $f$ is a mapping of $M$ into itself which has the property that for each $x \in M$ the limiting orbital diameter $r(x)$ of $f$ at $x$ is less than $\delta(O(x))$ when $\delta(O(x))>0$, then $f$ is said to have diminishing orbital diameters.

It is easy to give examples of nonexpansive mappings which have diminishing orbital diameters. For let $f: M \rightarrow M$ be such that for each $x \in M$ we have an $\alpha(x), 0 \leqq \alpha(x)<1$, and $d(f(x), f(y)) \leqq \alpha(x) d(x, y)$ for each $y \in M$. Thus, for $n>1, d\left(f(x), f^{n}(x)\right) \leqq \alpha(x) d\left(x, f^{n-1}(x)\right)$. This gives

$$
\begin{aligned}
\sup _{n} d\left(f(x), f^{n}(x)\right) & =\delta(O(f(x))) \leqq \sup _{n} \alpha(x) d\left(x, f^{n-1}(x)\right) \\
& =\alpha(x) \delta(O(x)) .
\end{aligned}
$$

Received by the editors March 7, 1967.

${ }^{1}$ Research supported by National Science Foundation Grant GP 5994. 
Hence,

$$
r(x)=\lim _{n \rightarrow \infty} \delta\left(O\left(f^{n}(x)\right)\right) \leqq \delta(O(f(x)) \leqq \alpha(x) \delta(O(x))<\delta(O(x)),
$$

if $0<\delta(O(x))$. Thus $f$ has diminishing orbital diameters.

For the type of mapping above, the existence of a fixed point yields the following. Let $f\left(x_{0}\right)=x_{0}$. Then $d\left(x_{0}, f(x)\right) \leqq \alpha\left(x_{0}\right) d\left(x_{0}, x\right)$. Also, $d\left(x_{0}, f^{n}(x)\right)=d\left(f\left(x_{0}\right), f^{n}(x)\right) \leqq \alpha\left(x_{0}\right) d\left(x_{0}, f^{n-1}(x)\right)$. Hence an induction argument shows that $d\left(x_{0}, f^{n}(x)\right) \leqq\left(\alpha\left(x_{0}\right)\right)^{n} d\left(x_{0}, x\right)$, for each $n \geqq 1$. Thus for any $x \in M$ we have $\lim _{n \rightarrow \infty} f^{n}(x)=x_{0}$.

THEOREM 1. Let $M$ be a metric space and let $f$ be a nonexpansive mapping of $M$ into itself which has diminishing orbital diameters. Suppose for some $x \in M$ a subsequence of the sequence $\left\{f^{n}(x)\right\}$ of iterates of $x$ has limit $z$. Then $\left\{f^{n}(x)\right\}$ has limit $z$ and $z$ is a fixed point of $f$.

Proof. Suppose $\lim _{k \rightarrow \infty} f^{n_{k}}(x)=z$. Then by a theorem of Edelstein $\left[5\right.$, Theorem $\left.1^{\prime}\right], z$ generates an isometric sequence. This means that for given positive integers $m$ and $n$,

$$
d\left(f^{m}(z), f^{n}(z)\right)=d\left(f^{m+k}(z), f^{n+k}(z)\right), \quad k=1,2, \cdots .
$$

Therefore if $k$ is any positive integer,

$$
\begin{aligned}
\delta(O(f(z))) & =\sup _{n \geqq 1} d\left(f(z), f^{n}(z)\right) \\
& =\sup _{n \geqq 1} d\left(f^{k}(z), f^{n+k-1}(z)\right) \\
& =\delta\left(O\left(f^{k}(z)\right)\right) .
\end{aligned}
$$

This implies

$$
\lim _{x \rightarrow \infty} \delta\left(O\left(f^{k}(z)\right)\right)=r(z)=\delta(O(f(z))) .
$$

But $r(z)=r(f(z))$. Since $r(f(z))=\delta(O(f(z)))$, the assumption that $f$ has diminishing orbital diameters enables us to conclude $\delta(O(f(z)))=0$ and thus $f(z)$ is a fixed point of $f$. Continuity of $f$ implies $\lim _{k \rightarrow \infty} f^{n_{k}+1}(x)$ $=f(z)$. Thus if $\epsilon>0$ there is an integer $k$ such that $d\left(f^{n_{k}+1}(x), f(z)\right)<\epsilon$. The fact that $f(z)$ is a fixed-point and $f$ is nonexpansive implies $d\left(f^{n}(x), f(z)\right)<\epsilon$ if $n \geqq n_{k}+1$. Thus $\lim _{n \rightarrow \infty} f^{n}(x)=f(z)$. But since a subsequence of $\left\{f^{n}(x)\right\}$ has limit $z, z=f(z)$ completing the proof.

CoROllary 1. If $M$ is any compact metric space and if $f$ is any nonexpansive mapping of $M$ into itself which has diminishing orbital diameters, then for each $x \in M$ the limiting orbital diameter $r(x)$ of $f$ at $x$ is zero, and the sequence $\left\{f^{n}(x)\right\}$ of iterates of $x$ converges to a fixed-point of $f$. 
3. Weakly compact sets. The concept of diminishing orbital diameters has significant implications in noncompact settings. In this section we obtain a result which implies that for closed convex subsets of a Banach space, weak compactness is sufficient to ensure the existence of a fixed-point for nonexpansive mappings with diminishing orbital diameters.

First we introduce some notation. Let $X$ be a Banach space. For a subset $A$ of $X, \mathrm{cl} \operatorname{co} A$ will denote the closed convex hull of $A$. For $x \in X$ and $\rho$ a positive number, $\mathcal{u}(x ; \rho)$ will denote the closed spherical ball centered at $x$ with radius $\rho: \mathcal{u}(x ; \rho)=\{z \in X:\|x-z\| \leqq \rho\}$.

THEOREM 2. Let $K$ be a bounded closed convex subset of a Banach space $X$, and let $M$ be a weakly compact subset of $X$. If $f$ is a nonexpansive mapping of $K$ into $K$ such that

(i) for each $x \in K, \mathrm{cl} \operatorname{co}(O(x)) \cap M \neq \varnothing$, and

(ii) $f$ has diminishing orbital diameters, then there is a point $x \in M$ such that $f(x)=x$.

Proof. If $\left\{K_{\alpha}\right\}$ is a descending chain of closed convex (hence weakly closed) subsets of $K$, each of which intersects $M$, then the weak compactness of $M$ implies $\left(\cap K_{\alpha}\right) \cap M \neq \varnothing$. Thus we may use Zorn's Lemma to obtain a subset $K_{1}$ of $K$ which is minimal with respect to being closed, convex, invariant under $f$, and having points in common with $M$. Let $M_{1}=K_{1} \cap M$.

Let $x \in K_{1}$ and suppose $\delta(O(x))>0$. We show this assumption leads to contradiction. By (ii) there is an integer $N$ such that

$$
\delta\left(O\left(f^{N}(x)\right)\right)=r<\delta(O(x)) .
$$

Let $U=\left\{z \in K_{1}:\left\|z-f^{n}(x)\right\| \leqq r\right.$ for almost all $\left.n\right\}$. Since $\delta\left(O\left(f^{N}(x)\right)\right) \leqq r$, $O\left(f^{N}(x)\right) \subseteq U$ and thus $U \neq \varnothing$. If $y \in U$ then for some integer $N_{1}$, $\left\|y-f^{n}(x)\right\| \leqq r$ if $n \geqq N_{1}$. Since $f$ is nonexpansive, $\left\|f(y)-f^{n+1}(x)\right\| \leqq r$ if $n+1 \geqq N_{1}+1$, and thus $U$ is mapped into itself. Clearly $U$ is convex since spherical balls of radius $r$ centered at each two points $u_{1}, u_{2}$ of $U$ contain some common set $O\left(f^{n}(x)\right)$. Thus a ball of radius $r$ centered at any point of the segment joining $u_{1}$ and $u_{2}$ will also contain $O\left(f^{n}(x)\right)$. Therefore, the closure $\bar{U}$ of $U$ is convex and mapped into itself by $f$; (i) implies $\bar{U} \cap M \neq \varnothing$, and the minimality of $K_{1}$ implies $\bar{U}=K_{1}$.

Let $p \in K_{1}$. Then since $p \in \bar{U}$, if $\epsilon>0$ there is a point $p^{\prime} \in U$ such that $\left\|p-p^{\prime}\right\|<\epsilon$. For some integer $N_{2},\left\|p^{\prime}-f^{n}(x)\right\| \leqq r$ if $n \geqq N_{2}$. Therefore $\left\|p-f^{n}(x)\right\| \leqq r+\epsilon$ if $n \geqq N_{2}$. Hence

$$
\text { cl co } O\left(f^{n}(x)\right) \subseteq \mathcal{u}(p ; r+\epsilon), \quad n \geqq N_{2} .
$$

By (i), cl co $O\left(f^{n}(x)\right) \cap M_{1} \neq \varnothing$, and since $M_{1}$ is weakly compact there is a point $t$ such that 


$$
t \in\left(\bigcap_{n=1}^{\infty} \operatorname{cl} \operatorname{co} O\left(f^{n}(x)\right)\right) \cap M_{1} .
$$

Then $t \in \mathcal{U}(p ; r+\epsilon)$ for each $\epsilon$. Thus $t \in \mathcal{U}(p ; r)$. Since this is true for each $p \in K_{1}$, it follows that

Therefore the set

$$
t \in \bigcap_{p \in K_{1}} u(p ; r) .
$$

$$
S=\left\{z \in K_{1}: K_{1} \subseteq u(z ; r)\right\}
$$

contains $t$, so $S$ is nonempty.

The remainder of our argument follows the argument given in [7].

It is easily seen that $S$ is closed and convex. Suppose for some $z \in S$, $f(z) \notin S$. Let $x \in H=\mathfrak{u}(f(z) ; r) \cap K_{1}$. Then $\|f(x)-f(z)\| \leqq\|x-z\|$ and $\|x-z\| \leqq r$. Because $f(z) \notin S$ by assumption, there is a point $x \in K_{1}$, such that $\|x-f(z)\|>r$. Hence $H$ is a proper subset of $K_{1}$. Since $H$ is closed, convex, and $H \cap M$ is nonempty (because $f(H) \subseteq H$ ), we have contradicted the minimality of $K_{1}$.

Therefore $f(S) \subseteq S$. But

$$
\delta(S) \leqq r=\delta\left(O\left(f^{N}(x)\right)\right)<\delta(O(x)) \leqq \delta\left(K_{1}\right)
$$

so $S$ is a proper subset of $K_{1}$. Again the minimality of $K_{1}$ is contradicted. Therefore the original assumption that $\delta(O(x))>0$ is incorrect, and $\delta(O(x))=0$. This implies $f(x)=x$.

Corollary 2. If $K$ is a closed, convex, weakly compact subset of $X$ and if $f$ is a nonexpansive mapping of $K$ into itself which has diminishing orbital diameters, then $f$ has a fixed point in $K$.

The above corollary is obtained by observing that since $K$ is weakly compact, condition (i) of the theorem holds trivially upon letting $M=K$.

It is not known whether Theorem 2 (or Corollary 2) remains true without the assumption of diminishing orbital diameters of $f$. This question is essentially equivalent to a question raised in [7] (as to whether the condition of "normal structure" is necessary for the theorem of [7]) which remains open. Similar results for nonexpansive mappings, without orbital constraints, are given by the authors in [1].

4. Normal structure. A very slight modification of the proof of Theorem 2 yields a theorem which is a generalization of Theorem 3 of $[1]$.

Let $A$ be a bounded subset of the Banach space $X$. A point $a \in A$ is a nondiametral point of $A$ if 


$$
\sup \{\|x-a\|: x \in A\}<\delta(A)
$$

A bounded convex subset $K$ of $X$ is said to have normal structure (Brodskii and Milman [3]) if for each subset $H$ of $K$ which contains more than one point there is a point $x \in H$ which is a nondiametral point of $H$.

TheOREM 3. Let $K$ be a bounded closed convex subset of a Banach space $X$, and let $M$ be a weakly compact subset of $K$. If $f$ is a nonexpansive mapping of $K$ into $K$ such that for each $x \in K$

(i) $\mathrm{cl} \operatorname{co}(O(x)) \cap M \neq \varnothing$, and

(ii) $\mathrm{cl} \operatorname{co}(O(x))$ has normal structure, then there is a point $x \in M$ such that $f(x)=x$.

Proof. Define $K_{1}$ as in the proof of Theorem 2 and obtain the set $U$ as follows: Suppose $\delta\left(K_{1}\right)>0$. Let $x \in K_{1}$. By (ii) there is a point $y \in \mathrm{cl} \operatorname{co}(O(x))$ such that

$$
\sup \{\|y-w\|: w \in \operatorname{cl} \operatorname{co}(O(x))\}=r<\delta(\operatorname{cl} \operatorname{co}(O(x))) .
$$

Let

$$
U=\left\{z \in K_{1}: O\left(f^{n}(x)\right) \subseteq u(x ; r) \text { for some } n\right\} .
$$

Then $y \in U$ so $U$ is not empty. The closure $\bar{U}$ of $U$ is convex and mapped into itself by $f$. Therefore $\bar{U}=K_{1}$. Following the argument of Theorem 2, one sees that the set

$$
S=\left\{z \in K_{1}: K_{1} \subseteq \mathfrak{u}(z ; r)\right\}
$$

is closed, convex, nonempty, and mapped into itself by $f$. But

$$
\delta(S) \leqq r<\delta(O(x)) \leqq \delta\left(K_{1}\right),
$$

so $S$ is a proper subset of $K_{1}$ contradicting the minimality of $K_{1}$. Therefore $\delta\left(K_{1}\right)=0$ and $K_{1}$ consists of a single point which is fixed under $f$.

Since compact convex sets have normal structure (this is essentially Lemma 1 of [4]), we have the following corollary.

CoROllary 3. If $K$ is a closed convex weakly compact subset of $X$ and if $f$ is a nonexpansive mapping of $K$ into $K$ for which $O(x)$ is precompact for each $x \in K$, then $f$ has a fixed-point in $K$.

Precompactness of $O(x)$ does not in general imply $f$ has diminishing orbital diameters. In fact, as a consequence of the above corollary, one might note that if $f$ is a periodic isometry of $K$ into $K, f$ has a fixed-point. 
Some examples of spaces which possess normal structure are given in $[2]$.

\section{REFERENCES}

1. L. P. Belluce and W. A. Kirk, Fixed-point theorems for families of contraction mappings, Pacific J. Math. 19 (1966), 213-217.

2. L. P. Belluce, W. A. Kirk and E. F. Steiner, Normal structure in Banach spaces, Pacific J. Math. (to appear).

3. M. S. Brodskii and D. P. Milman, On the center of a convex set, Dokl. Akad. Nauk SSSR 59 (1948), 837-840.

4. Ralph De Marr, Common fixed-points for commuting contraction mappings, Pacific J. Math. 13 (1963), 1139-1141.

5. M. Edelstein, On fixed and periodic points under contractive mappings, J. London Math. Soc. 37 (1962), 74-79.

6. - On nonexpensive mappings, Proc. Amer. Math. Soc. 15 (1964), 689-695.

7. W. A. Kirk, $A$ fixed point theorem for mappings which do not increase distances, Amer. Math. Monthly 73 (1965), 1004-1006.

University of California, Riverside 